\title{
7 Ein integrales Modell des Bewusstseins
}

\author{
Katharina Ceming und Michael Habecker
}

\section{Vier Perspektiven auf das Bewusstsein}

Ken Wilbers Verdienst im Kontext der Erforschung des Bewusstseins besteht darin, dass er ein sehr umfangreiches Modell entwickelt hat, in dem er vier strukturell verschiedene Zugangsweisen zur Wirklichkeit aufzeigt. Jede dieser Zugangsweisen, die einem Quadranten in seinem Modell entspricht (dazu gleich mehr), bietet andere Einsichten und Erkenntnisse über die Wirklichkeit. Die Frage nach dem, was Bewusstsein ist, wird maßgeblich von der Perspektive des jeweiligen Quadranten mitbeeinflusst. Die integrale Theorie und Praxis würdigt sie alle (s. Abb. 12).

\section{INDIVIDUELL}

\begin{tabular}{|c|c|}
\hline \begin{tabular}{l}
\multicolumn{1}{c}{ ICH } \\
Innerpsychisch \\
Phänomenologisch \\
Strukturell \\
Psychodynamisch
\end{tabular} & \begin{tabular}{l}
\multicolumn{1}{c}{ ES } \\
Physiologisch \\
Verhaltensorientiert \\
Reiz-Reaktion
\end{tabular} \\
\hline \begin{tabular}{l}
\multicolumn{1}{c}{ WIR } \\
Inter-subjektiv \\
Kollektiv \\
Gruppendynamisch \\
Beziehungsorientiert
\end{tabular} & $\begin{array}{l}\text { SIE } \\
\text { Inter-objektiv } \\
\text { Systemisch } \\
\text { Prozesshaft } \\
\text { Funktional }\end{array}$ \\
\hline
\end{tabular}


Durch die individuell/innerliche Perspektive des linken oberen Quadranten erkenne ICH Bewusstsein unmittelbar in mir selbst (Die Ichform wird hier bewusst zur Beschreibung des oberen linken Quadranten verwendet.). Dies können Sie in diesem und jedem Augenblick Ihres Lebens durch eine einfache Innenschau unmittelbar überprüfen. Bewusstsein erschließt sich direkt durch die Wahrnehmung von allem, was in mir passiert. Ich kann Müdigkeit, Freude, Langeweile usw. direkt in mir wahrnehmen. Eine typische Aussage aus dieser Erkenntnisperspektive heraus lautet: „die Welt entsteht in meinem Bewusstsein“. Die daraus erwachsende Weltsicht tendiert zu einem Idealismus und Subjektivismus, wenn sie verabsolutiert wird.

Doch der Geist ist nicht nur in Bewusstseinsinhalten durch die persönliche Innenschau zu erfahren, sondern er hat auch ein körperliches Korrelat. Im oberen rechten Quadranten erforschen Verhaltenspsychologen, Biologen, Neurologen und Mediziner was im menschlichen Gehirn passiert, wenn der Mensch sich freut, sich ärgert, wenn er denkt, hofft, Pläne verfolgt, etwas tut, oder liebt usw. Im oberen linken Quadranten liebe ICH. Im oberen rechten, dem ES-Quadranten, kann man das Individuum von außen beobachten und untersuchen. Man kann sein Verhalten sehen, seine Blutwerte messen, ein Kardiogramm oder einen Gehirnscan machen. Ein Arzt kann bei einem verliebten Patienten vielleicht einen veränderten Dopamin- und SerotoninSpiegel messen, da das Clücksgefühl, dass ICH erlebe, im Körper mit einem veränderten Hormonwert korreliert. Dass der Grund dafür ein Verliebtsein ist, weiß der Arzt aber nur, wenn es ihm sein Patient sagt. Nun hat der Arzt zusätzlich zu seinen objektiven Messergebnissen eine individuelle und innere Perspektive - und beide Erkenntnisse sind nicht aufeinander reduzierbar. Werden die Erkenntnisse dieses rechten oberen Quadranten jedoch verabsolutiert, erhält man Aussagen wie die des Behavioristen John. B. Watson:

„Mit aller Wahrscheinlichkeit kann man die restlichen Probleme [der Psychologie] noch so umformulieren, dassfeinere Methoden der Verhaltensforschung (die sicher kommen müssen) zu ihrer Lösung führen.“(Watson 1997, S. 28)

Für Watson existiert nur die ES-Wirklichkeit. Alles, was ICH erlebe, ist dieser Ansicht nach nur ein „Abfallprodukt“ dessen, was im ES-Bereich passiert.

Doch mit den beiden individuellen, oberen Quadranten haben wir erst die Hälfte der Wirklichkeit in den Blick genommen, denn kein Individuum ist eine Insel und kein Bewusstsein ist ausschließlich etwas Privates. Jeder Mensch lebt immer auch in Beziehungen, in einem größeren Gesamten. Er ist Teil eines Systems, das nach eigenen Regeln und Gesetzmäßigkeiten agiert. Die Außenseite dieser kollektiven Dimension wird durch den unteren rechten Quadranten, den SIE-Quadranten repräsentiert. Niklas Luhmann, Vater der Systemtheorie, kommt daher, aus einer kollektiv-systemischen Perspektive heraus betrachtet, zu einer ganz anderen Sicht des Bewusstseins:

\section{„So haben soziale Systeme keine Verwendung für Bewußtsein, .... (Luhmann 1987, S. 60)}

Diese Blickrichtung zielt auf sich selbst aufrechterhaltende „autopoietische“ Systeme, mit all ihren Auswirkungen auf das individuelle Bewusstsein. Ein berühmter Pionier dieser Perspektive ist Karl Marx, der den legendären Satz äußerte:

„Esist nicht das Bewusstsein der Menschen, dasihr Sein, sondern umgekehrt ihr gesellschaftliches Sein, dasihr Bewusstsein bestimmt." (Marx) 
In diesem Satz vereinigen sich sowohl die Größe als auch die Grenze der systemischen Perspektive auf den Menschen. Es ist unbestritten, dass die Systeme, in denen wir leben (Wirtschaft, Finanzen, Infrastruktur, Ökologie, Energie, Politik, Bildung, Internet usw.) nicht nur unser Verhalten, sondern auch unser Bewusstsein mitbestimmen und formen. Aber sie sind nicht der einzige und auch nicht der kausale Bestimmungsfaktor, sondern im Bild der Quadranten gesprochen, nur einer von vier Quadranten. Alle Versuche, durch Systemveränderungen alleine den „neuen Menschen“ zu schaffen, waren bisher, vorsichtig formuliert, nicht erfolgreich.

Schließlich bleibt noch die Perspektive des unteren linken Quadranten. Hier dominiert die Sicht auf das, was WIR als kollektive Innerlichkeit und Bewusstheit bezeichnen. Ende des 19. Jahrhunderts sprach man von der Völkerpsychologie (ein Buchtitel von Wilhelm Wundt) oder von der Psychologie der Massen, so das gleichnamige Werk von Gustave Le Bon (2009). Die grundlegende Frage, die hier gestellt wird, lautet: Welche Prozesse ereignen sich in Gruppen und wie fühlen sich diese gemeinschaftlich an. Ethnologie, Kulturwissenschaften, um nur einige Forschungsdisziplinen zu nennen, die in diesem Bereich aktiv sind, versuchen, Massenphänomene wie Gefolgschaft, Rebellion, Fankult, aber auch kollektive Stimmungswandel oder Wählerverhalten von innen heraus zu erklären.

Eine integrale Theorie des Bewusstseins wird alle vier genannten Forschungsrichtungen in ihrer Betrachtungsweise von Bewusstsein würdigen, diese als unterschiedliche Perspektiven auf ein Thema und Phänomen nutzen, und deren Ergebnisse in einem Gesamtbild zusammenführen. In diesem Bild findet jede der vier Erkenntnisperspektiven ihre Bedeutung und Würdigung. Die integrale Theorie ermöglicht es aber auch, die Grenzen der jeweiligen Perspektiven zu erkennen und sie vermeidet damit Ismen wie:

n Ideal-ismus „Der Geist bestimmt die Materie.“

- Material-ismus „Materie allein ist real.“

- System-ismus „Das individuelle Bewusstsein ist systemisch determiniert.“

- Konstruktiv-ismus „Individualität ist eine Illusion, weil der Menschen kulturell konstruiert und bestimmt ist.“

- Individual-ismus und Subjektivismus „Mein Bewusstsein, in dem alles andere erscheint, ist das einzig Reale.“

- Biolog-ismus „Der Mensch ist seine Biologie (seine Gene, sein sich selbst organisierendes Gehirn)“ (oder: „Der Mensch ist was er isst.“)

- (Quanten)physikal-ismus „Die Quantenwirklichkeiten sind der wahre bestimmende Faktor“

\section{Es gibt ein Innen}

Irgendwann vor sehr langer Zeit muss es passiert sein: Ein Mensch oder ein früher Vorfahre des Menschen wurde sich zum ersten Mal, wenn auch nur ganz vage und unbestimmt, seiner eigenen Innerlichkeit bewusst, ohne dieses Gefühl, diese Wahrnehmung jedoch schon formulieren zu können. Auch in der individuellen Biografie und Entwicklung eines jeden Menschen gibt es einen Punkt, wo er oder sie sich zum ersten Mal selbst-bewusst wird, im Sinne von „ich bin und erlebe mich als ein empfindendes Wesen“. Dies ist sowohl individuell als auch kollektiv betrachtet ein großer und großartiger Augenblick. 
Wenn heute dieser Bereich der inneren, erlebten Bewusstwerdung, die ein Phänomen des oberen linken Quadranten ist, oftmals nur als ein Nebenprodukt materieller Vorgänge betrachtet wird, zeigt sich darin sehr eindrucksvoll der Reduktionismus des rechten oberen Quadranten, der alle Erlebnisgehalte auf materielle Prozesse reduzieren möchte. Genau diese Erlebnisinhalte des Bewusstseins, die als Qualia bezeichnet werden, können aber von allen Theorien, die im rechten oberen Quadranten ihren Sitz haben naturgemäß nicht erklärt werden. Und so sind bis heute die Qualia der Stein des Anstoßes in allen materialistischen Bewusstseinstheorien. Wenn wir das integrale Paradigma zugrunde legen, dann wird verständlich, weshalb materialistische Theorien, die wichtig und bedeutsam sind, niemals die Herkunft der Qualias erklären können, sowenig die genauesten phänomenologischen Beobachtungen unseres Erlebens im linken oberen Quadranten uns nichts über die neurophysiologischen Vorgänge in unserem Gehirn sagen.

Im Folgenden möchten wir uns etwas näher mit dem linken oberen Quadranten beschäftigen, da das ICH dort nicht nur eine schier unerschöpfliche Anzahl von verschiedenen Bewusstseinsinhalten kreiert und erfährt, sondern wir bei der Untersuchung dieses ICH-Bereichs auch Strukturen und Dynamiken des Bewusstseins antreffen können, die alle ins Spektrum dessen gehören, was zum Bewusstsein zählt. Um diese zu erkennen, benötigen wir jedoch andere Methodiken und Untersuchungswerkzeuge als bei der Erkenntnis der Inhalte.

\section{Inhalte, Strukturen und Dynamiken des Bewusstseins}

\section{Inhalte des Bewusstseins}

Einem Vorschlag von Ken Wilber folgend, lassen sich hinsichtlich des Bewusstseinsbegriffes mindestens drei nicht aufeinander reduzierbare Hauptaspekte unterscheiden:

- Inhalte,

- Strukturen und

- Dynamiken des Bewusstseins.

Die Inhalte (oder Phänomene) des Bewusstseins sind das, worauf man als erstes stößt, wenn man eine Innenschau vollzieht. Manches wird körperlich-somatisch, anderes emotional oder auch gedanklich erlebt. Realität hat es für das Ich sobald es als Bewusstseinsinhalt bewusst erlebt wird. Solche Inhalte können sein:

Abscheu, Aggression, Angst, Bedrückung, Begeisterung, Druck, Eifersucht, Einsamkeit, Einssein, Ekel, Enttäuschung, Freude, Furcht, Gier, Glück, Glückseligkeit, Hass, Heiterkeit, Hunger, Jucken, Kälte, Leid, Liebe, Lust, Nervosität, Reue, Schadenfreude, Scham, Schmerz, Schuld, Sorge, Stärke, Stille, Übelkeit, Verlegenheit, Vertrauen, Verwirrung, Wut, Zuneigung, Zuversicht, Wärme ...

Bestimmte Erlebnisinhalte wie Einssein, Glückseligkeit, Leerheit können als religiöse oder spirituelle Inhalte bezeichnet werden. Die Wahrnehmung und „Untersuchung “ dieser Inhalte dürfte vermutlich die älteste Form der Bewusstseinsphänomenologie sein, die bereits in frühen schamanischen Kulturen stattfand. Die in der Geschichte der Menschheit am häufigsten erprobte Form des Kennenlernens, Erlebens und „Untersuchens“ dieser Bewusstseinsinhalte ist die Innenschau, Introspektion, Meditation oder Kontemplation. 


\section{Vertikale Strukturen des Bewusstseins}

Weit weniger offensichtlich, wenngleich enorm wirksam und bedeutend, ist die zweite der genannten Kategorien, die der Bewusstseinsstrukturen. Während Bewusstseinshalte kommen und gehen, sind (innere) Strukturen das, womit wir die Inhalte unserer Wahrnehmung interpretieren und ihnen einen Kontext geben. Sie entwickeln sich im Laufe unseres Lebens und sind relativ stabil. Wenn ein Mensch einmal eine bestimmte Ebene/Stufe/Struktur erlangt habe, fällt er nicht mehr auf eine tiefere Stufe zurück, es sei denn, es passiert etwas auf der neuronalen Ebene, z.B. er wird dement, hat eine Hirnverletzung, einen Schlaganfall etc. So wie jeder Einzelne von uns von der Geburt bis hin zum Erwachsenwerden bestimmte Entwicklungsstufen seines Bewusstseins durchlaufen hat, so hat sich vermutlich auch die Menschheit entwickelt. Wichtig ist zu erkennen, dass sich mit jeder Entwicklungsstufe des Bewusstseins auch die Sichtweisen auf die Wirklichkeit und die menschlichen Werte verändern.

Ein Wissenschaftler, der sich besonders mit dem Phänomen der Bewusstseinsstrukturen und Werteentwicklung beschäftigte, war der amerikanische Psychologe Clare Graves (http://www.clarewgraves.com/store.html - abgerufen am 16.9.2013)

Graves erkannte in seinen Studien, dass jede Bewusstseinsstufe, eine bestimmte Weltsicht und damit auch bestimmte Werte vertritt. In seinem Entwicklungsmodell sind alle aufeinanderfolgenden Stufen in einem dynamischen System organisiert. Aus diesem Grund nannten seine Nachfolger Don Beck und Chris Cowan ihr Modell Spiral Dynamics (Beck 2008) Jede Stufe der Entwicklung steht in unmittelbarem Bezug zu vorausgegangenen oder nachfolgenden Stufen - in einem Verhältnis von „Transzendieren und Bewahren“. Die Entwicklungsstufen des individuellen Bewusstseins spiegeln sich in der Kulturgeschichte der Menschheit.

\section{Bislang sind acht bzw. neun verschiedene Bewusstseinsstufen und damit ver- bunden auch acht bzw. neun Werteebenen aufgetaucht.}

Graves war überzeugt, dass in Zukunft noch weitere Stufen emergieren werden. Um sein System etwas zu vereinfachen, belegten die Macher von Spiral Dynamics jede Stufe mit einem Farbwert.

Auf der untersten Ebene, der beigen, geht es um das pure instinktive Überleben. Werte spielen hier noch keine Rolle. Wir befinden uns in einer Zeit vor etwa 100.00o Jahren.

Erst als das Überleben gesichert war, konnte etwa $50.000 \mathrm{v}$. Chr. die purpurne, die magisch/animistische Werteebene entstehen. Sie ist von der Wahrnehmung und der Verbundenheit mit mythischen Geistwesen, Ahnen und dem eigenen Stamm bestimmt. Die entscheidende Handlungsmotivation ist die Herstellung von Sicherheit, was nur gelingt, wenn die Geistwesen und Ahnen zufriedengestellt werden. Der Mensch ist hier noch Teil des Kollektivs.

Etwa gegen 7.0oo v. Chr. begannen Einzelne aus dem Sicherheit stiftenden Verband auszubrechen und sich mit Gewalt an die Spitze der jeweiligen Cruppe zu setzen. Beck kennzeichnet diese Phase des Aufbruchs eines impulsiven, egozentrischen Bewusstseins, das seine eigenen Interessen gewaltsam durchsetzt, mit der Farbe Rot. Das beherrschende Thema ist nicht mehr Überleben oder Sicherheit, sondern das eigenmächtige Handeln. 
Da diese Phase durch eine große Willkür und Gewalt geprägt war, begann sich ab etwa $3000 \mathrm{v}$. Chr. eine neue Ebene bemerkbar zu machen, die blauen Stufe. Sie ist bestimmt von der Überzeugung, dass es eine höhere Ordnung gibt, die dem Leben Sinn und Richtung gibt, weshalb die Befolgung von Ordnung gebenden Regeln absolut notwendig ist. Es gibt unveränderliche Prinzipien von ,richtig' und ,falsch'. Wer sie befolgt, wird von Gott oder den Göttern belohnt, wer sie missachtet, wird bestraft. Der Mensch ist in ein straffes religiöses und soziales Unterordnungssystem eingepasst. Das Weltbild ist dualistisch (richtig-falsch, gut-böse, männlich-weiblich, Freund-Feind, Erlösung-Verdammung, entweder-oder). Hier kommt zum ersten Mal so etwas wie Ethik ins Spiel, allerdings ist sie noch ethnozentrisch, d.h. auf das eigene Volk oder die Anhänger der eigenen Religion bezogen.

Mit der orangenen Ebene, die durch Rationalität gekennzeichnet ist und in welcher die Technik und Naturwissenschaften zu ihrer Blüte kamen, bildete sich in der Neuzeit eine neue Stufe heraus. Der Einzelne begann sich aus den hierarchischen Strukturen zu befreien. Die Devise dieser Ebene könnte lauten: Jeder ist seines eigenen Glückes Schmied. Die Welt und alles in ihr wird als gut geölter Mechanismus betrachtet, der nach eigenen Vorstellungen verändert werden kann. Erfolg und Machbarkeit sind wesentliche Motivationen des Handelns. Mit den negativen Seiten dieser Ebene sind wir heute weltweit konfrontiert: Ressourcenverschwendung, ökologische Katastrophe etc. Positiv an dieser Ebene ist allerdings, dass hier die Grundfeste einer universellen Ethik gelegt ist, da nicht mehr die Zugehörigkeit zur eigenen Sippe oder Religion im Vordergrund steht.

Quasi als Kritik an dieser materialistisch, mechanistisch, rationalistischen Weltsicht entwickelte sich ein neues Werteparadigma, das Beck und Cowan grün gekennzeichnet haben, und das von seiner Ausrichtung egalitär, multiperspektivisch und relativistisch ist und die Verbundenheit aller mit allen und allem ins Zentrum des Handelns stellt. Oberste ethische Priorität hat die Sorge Füreinander (Ceming 2012, S. 20-21). Grün ist ferner gekennzeichnet vom Wunsch, niemanden und nichts zu benachteiligen, weshalb grün sich auch gegen jede Form von Wertungen und Bewertungen verwehrt. Grün möchte, dass alle Positionen gleichermaßen berücksichtigt werden, weil aus grüner Perspektive Hierarchien stets Ausdruck von Machtmissbrauch sind. Hier wird jedoch das Grundproblem von grün deutlich. Durch diese Haltung werden auch Positionen gefördert, die auf die Abschaffung genau dieser Werte zielen.

Besonders deutlich wird dies in der aktuellen Debatte wie unsere Gesellschaft mit extremistischen und fundamentalistischen Gruppierungen umgehen soll, deren Ideologien in rot und blau gründen.

n Gilt das Recht auf Meinungs- und Religionsfreiheit auch für sie?

- Dürfen Nazis ihre volksverhetzenden, rassistischen Parolen unter Rückgriff auf die Meinungsfreiheit überall frei verkünden und dafür werben, obwohl ihre Ideologie genau dieses Recht für alle ja aufheben möchte?

- Dürfen fundamentalistische religiöse Cruppierungen, welche die Unterordnung von Frauen, die Abschaffung der Demokratie, die Etablierung eines Cottesstaates fordern, dies unter dem Mantel der Religions- und Meinungsfreiheit tun?

Grün hat darauf keine Antworten, weil diese Ebene nicht zwischen echten und machtbedingten Hierarchien unterscheiden kann. Eine Lösung bringt erst die nächste Ebene. 
Graves erkannte im Übergang von grün nach gelb einen qualitativen Sprung. Erst die gelbe Ebene ist in der Lage, den Wert und die Berechtigung aller vorausgegangenen Ebenen wahrzunehmen und gleichzeitig zu erkennen, dass mit jeder Ebene, die sich entwickelte ein größeres Potenzial von Bewusstwerdung entstand. Gelb hat die Fähigkeit, Positionen zu verbinden, komplex zu denken, Erkenntnis- und Wertehierarchien anzuerkennen und falsche Hierarchien zu entlarven. Gelb wird akzeptieren, dass ein Mensch aus welchen Gründen auch immer nicht bereit ist, anzuerkennen, dass alle Menschen gleichwertig sind, aber gelb wird nicht zulassen, dass diese sexistischen oder rassistischen Werte im öffentlichen Raum eingefordert und gelebt werden.

Die türkise Ebene ist durch ein holistisches, intuitives Denken und kooperatives Handeln gekennzeichnet. Sie beginnt sich jedoch gerade erst zu entwickeln.

Wenn man die Ergebnisse hinsichtlich der Werteentwicklung von Graves und seinen Nachfolgern noch zusammenfassen will, so kann man sagen, die Entwicklung läuft von egozentrisch zu ethno-/soziozentrisch bis hin zu weltzentrisch. Als Kleinkinder agieren wir egozentrisch. Wir können uns noch nicht in andere hineinversetzen, und daher sind unsere eigenen Bedürfnisse das, woran wir uns ausrichten. Im Laufe unserer Sozialisierung lernen wir dann, uns in einer Gemeinschaft zurechtzufinden, und die Ethik dieser Gemeinschaft verinnerlichen wir, als eine soziozentrische Entwicklungsstufe.

Erst in einem weiteren Entwicklungsschritt sind wir in der Lage, die Begrenztheit dieser Werte, die andere, die außerhalb der eigenen Gemeinschaft leben, nicht berücksichtigt, zu erkennen. Nun können wir eine weltzentrische Haltung entwickeln, die gleiche Rechten, aber auch Verpflichtungen für alle Menschen beinhaltet.

\section{Horizontale Strukturen}

Neben der vertikalen sich entwickelnden Strukturiertheit unseres Bewusstseins gibt es auch horizontale Strukturen, die meist in typologischen Modellen ihren Ausdruck finden. Eine Introvertiertheit ist nicht besser oder schlechter als eine Extrovertiertheit, sondern anders, und dieses Kriterium unterscheidet horizontale Strukturen, die man meist als Typologien bezeichnet, von vertikalen wie z.B. Werten im Bewusstsein. Die entscheidende, und nur geisteswissenschaftlich zu beantwortende Frage dabei ist: Was sind horizontale Strukturen bzw. Typologien und was sind vertikale Strukturen?

Klassische typologische Formen des Bewusstseins sind z.B. Charaktereigenschaften, wobei es hier passieren kann, dass durch die bewusstseinsmäßige Verortung einer Kultur ihr ihrer damit verbunden Werte (Quadrant unten links) bestimmte Charaktereigenschaften besser oder schlechter bewertet werden. Eine Kriegerkultur bewertet Mut und Aggression positiver als eine Kultur, in der Sanftheit und Freundlichkeit Leitwerte sind. Aber auch Persönlichkeitsentwicklungssysteme wie das Enneagramm arbeiten mit Typologien. Eine enneagrammatische Vier-Fixierung ist nicht schlechter oder besser als eine Fünferfixierung. Eine erlöste Fünf ist hingegen weiter als eine unerlöste Fünf, da sie ihre Potenziale und Probleme erkannt hat und an Lösungen arbeitet. Hier fand eine vertikale Entwicklung statt.

Im Kontext einer integralen und globalen (Werte-)Gemeinschaft ist es jedoch unabdingbar horizontale und vertikale Strukturen zu unterscheiden. Wenn aus echten (d.h. gleichwertigen) Typen wie Hautfarbe, Geschlecht oder Sprachen Hierarchien 
gemacht werden, dann entstehen Rassismen und Unterdrückung. Diese Art der falschen Hierarchisierung hat die Kulturgeschichte der Menschheit Jahrtausende lang geprägt, und das nicht nur im Westen, wie viele glauben. Der Westen war lediglich im internationalen Vergleich darin am „erfolgreichsten“. Wenn jedoch aus echten Wertestufen Typen gemacht werden, dann ist auf einmal die Haltung von echtem Mitgefühl nicht mehr besser als eine Haltung, die ohne zu zögern bereit ist, einen anderen Menschen zu töten. Das Ergebnis dieser Verwechslung ist ein falscher Egalitarismus und eine Werteindifferenz.

\section{Bewusstseinsdynamiken}

Die dritte der genannten Bewusstseinskategorien, die Bewusstseinsdynamiken, verweisen darauf, dass die Inhalte unseres Bewusstseins nicht einfach nur herumliegen und darauf warten, von den Hintergrundstrukturen interpretiert zu werden, sondern dass eine enorme Fülle von Wechselwirkungen (meist unbewusst) im Bewusstseins vonstattengeht, die wir generell als Psychodynamik bezeichnen können. Auch hierfür gibt es eine eigene Sprache, mit Begriffen wie:

Abspaltung, Adaption, Fehlleistung, Fixierung, Hemmung, Kompensation, Komplex, Neurose, Phobie, Projektion, Psychose, Regression, Sublimierung, Suggestion, Tabu, Trauma, Übertragung, Verdichtung, Verschiebung, Wahn, Zwang ...

Unter der Überschrift „kognitive Verzerrungen“ finden sich in der Literatur lange Listen von verschiedensten Möglichkeiten, wie wir uns selber psychologisch täuschen können, als eine Art geistige Ergänzung zu der Vielzahl von optischen Täuschungen.

Auch diese Erkenntnisse sind schon lange intuitiv erspürt und beschrieben worden, obwohl deren systematische Erforschung erst um das Jahr 1900 mit Wissenschaftlern wie Siegmund Freud und C.G. Jung begann. Das biblische Gleichnis vom „Splitter“ im Auge des anderen, der eigentlich nur eine Reflektion des „Balkens“ im eigenen Auge ist (Matthäus 7,3), ist eine frühe und geniale Intuition der Verdrängungs- und Projektionsdynamik. Aus einer ähnlich frühen Zeit stammt die Empfehlung der Wüstenväter „lasse die Sonne nicht über deinen Zorn untergehen“. Wer seinen eigenen Zorn nicht anschaut und das damit verbundene destruktive Potenzial nicht rechtzeitig entschärft, läuft Gefahr, von diesen Emotionen überschwemmt zu werden. Die Erkennung dieser Psychodynamiken stellt einen enorm wichtigen Beitrag zur Bewusstwerdung dar.

\section{Zu guter Letzt}

Es wurde ein langer Weg sowohl individuell als auch kollektiv zurückgelegt, von einer ersten und vagen Differenzierung gegenüber der eigenen Innerlichkeit, bis zu unserem heutigen Wissens- und Erkenntnisstand dem Thema Bewusstsein gegenüber. Dies betrifft aber nicht nur das Wissen über das Bewusstsein, sondern auch die zunehmende Bewusstheit über uns selbst, unsere Mitmenschen und die äußere Welt.

Eine integrale Theorie wird zum einen die Historie dieses langen Weges zu würdigen wissen sowie die, auf diesem Weg gewonnenen Erkenntnisschätze. Sie hilft, Inhalte und Strukturen (vertikal und horizontal) sowie Dynamiken des Bewusstseins zu 
unterscheiden. Sie erkennt, welche Methoden zur Bearbeitung welche Fragen und Inhalte hilfreich sind. Neben den Geisteswissenschaften als den natürlichen Verwandten der Bewusstseinsforschung wird eine integrale Theorie dazu auch die Natur- und Systemwissenschaften einbeziehen, in ihrer unverzichtbaren Außenperspektive auf das, was Menschen individuell und kollektiv als Bewusstsein erleben. Und schließlich wird eine integrale Theorie nicht nur eine Theorie bleiben, sondern immer auch den Praxisaspekt unterstützen und vorantreiben sowohl individuell als auch kollektiv, indem sie konkrete Fragestellungen aufwirft:

- Wie können Menschen individuell und kollektiv (mehr) Zugang zu den Inhalten ihres Bewusstseins erlangen?

- Wie lässt sich Bewusstsein entwickeln?

- Wie lassen sich individuelle und kollektive Bewusstseinsstrukturen und -dynamiken erkennen, aus denen heraus Menschen fühlen, denken, sprechen und handeln?

- Was ist besser (im Sinne von weiter entwickelt) und was ist anders (im Sinne von alternativ)?

- In was für einer Welt wollen wir leben?

- Mit welchen Werten als Hintergrundstrukturen wollen wir Zusammenleben organisieren?

- Worin gründet meine Interpretation von Wirklichkeit und worin gründen meine und unsere Werte?

- Was kann auf dem (Bewusstseins-)Entwicklungsweg alles schief gehen und wie lässt sich das, was schiefgelaufen ist, heilen?

Und schlussendlich die Frage, die viele spirituelle und philosophischen Systeme stellen:

Was wäre, wenn alle Formen, die äußerlich und innerlich erfahrbar sind, nur vergänglich sind? Gibt es etwas, das zeitlos ist, weil alle Zeit daraus entsteht, etwas, das unendlich ist, weil aller Raum darin enthalten ist, etwas, das frei von Gestaltungen ist, weil alle Formen daraus hervorgehen? Und was würde dies für unser Menschsein bedeuten?

\section{Literatur}

Beck D (2008) Spiral Dynamics. Leadership, Werte und Wandel. Kamphausen Bielefeld 2. Auflage Ceming K (2012) Spiritualität im 21. Jahrhundert. Phänomen Verlag, Hamburg

Habecker M u. Student S (2011) Wissen, Weisheit, Wirklichkeit: Perspektiven einer aufgeklärten Spiritualität. Kamphausen Verlag, Bielefeld

Le Bon, G (2009) Psychologie der Massen. Nikol Verlags-GmbH

Luhmann N (1987) Soziale Systeme. Suhrkamp Verlag, Frankfurt a.M.

Marx K (Internetausgabe) Vorwort Zur Kritik der politischen Ökonomie, Marx K \& Engels F Werke. Dietz Verlag, Berlin http://kulturkritik.net/systematik/philosophie/mew/pop_mew.php?bd=13\&pg=9 (abgerufen am 20.6.2013)

Watson I (1997) Behaviorismus. Klotz Verlag, Eschborn bei Frankfurt. 4. unveränderte Auflage

Wilber K (2005) Das Wahre, Schöne, Gute. Fischer Verlag, Frankfurt a.M.

Wilber K (2006) Eros, Kosmos, Logos. Fischer Verlag, Frankfurt a.M. 4. Auflage

Wilber K (2007) Integrale Spiritualität. Kösel Verlag, München 2. Auflage

Wilber K u. Engler I u. Brown D (1988) Psychologie der Befreiung. Arbor Verlag, Bern, München, Wien 


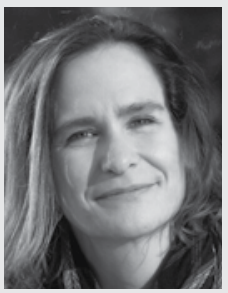

apl. Prof. Dr. Dr. Katharina Ceming

Nach ihrem Studium der katholischen Theologie und Germanistik an der Universität Augsburg und einer Promotion im Fach Philosophie, habilitierte sie 2002 in Fundamentaltheologie. Im Anschluss an eine dreijährige Professorentätigkeit an der Universität Paderborn erwarb sie im Jahr 2009 mit einer Arbeit über das Verhältnis von Menschenrechten und Religionen noch einen theologischen Doktortitel. Katharina Ceming beschäftigt sich seit vielen Jahren neben den religiösen, philosophischen, kulturellen und sozialen Aspekten der Weltreligionen besonders mit Mystik und Spiritualität sowie der Integralen Theorie Ken Wilbers. Im April 2008 erhielt sie den Mystikpreis der Theophrastus Stiftung. Zahlreiche Publikationen.

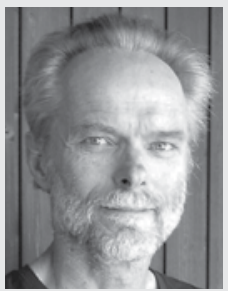

\section{Michael Habecker}

Beschäftigung mit Ken Wilbers Werk in Theorie und Praxis seit 1985. Mitbegründer des deutschsprachigen Integralen Forums e.V. (www.integralesforum.org) und Referent und Seminarleiter zu integralen Themen. Autor des Buches „Ken Wilber - eine integrale (R)EVOLUTION“ und Co-Autor des Buches „Wissen, Weisheit, Wirklichkeit“. 